\title{
Plan Especial de Protección de Infraestructura Verde y Biodiversidad de Salamanca 2020-35 (PEPIVB) como estrategia de conservación de sus valores universales excepcionales: naturales y patrimoniales
}

\section{Beatriz Diosdado Calvo ${ }^{a}$ y Carlos Macarro Alcalde}

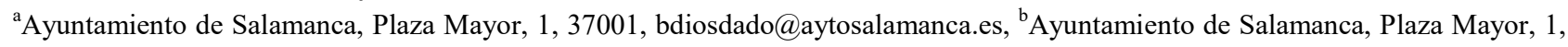
37001, cmacarro@aytosalamanca.es.

\begin{abstract}
Resumen
Transcurridos treinta años de la inclusión de Salamanca en la Lista del Patrimonio Mundial de Unesco, se ha producido una evolución en los criterios de gestión de los bienes culturales que obliga a una nueva comprensión del valor del sitio, desde el punto de vista cultural y medioambiental. En este contexto, el Ayuntamiento de Salamanca acaba de aprobar el Plan Especial de Protección de Infraestructura Verde y Biodiversidad 2020-35 con el objeto de crear una estrategia que integre holísticamente los valores naturales, sociales, económicos y patrimoniales para que configuren un territorio preparado para afrontar los nuevos desafios de las ciudades a través de soluciones basadas en la naturaleza.

La estrategia se concibe como un "plan vivo, en evolución continua y con capacidad de adaptación a los requerimientos de la ciudad" que apuesta por dotarse de una figura urbanística como herramienta de consolidación (ésto constituye una novedad con respecto a otras ciudades verdes). Otra característica es la definición de su ambito que abarca la totalidad de su témino, a la vez que deja apuntadas las soluciones con términos colindantes y la malla regional. Es una estrategia innovadora que utiliza como base la infraestructura verde junto con otro valor universal: su patrimonio.
\end{abstract}

Palabras clave: infraestructura verde urbana, patrimonio mundial, soluciones basadas en la naturaleza, malla verde urbana, cambio climático, servicios ecosistémicos, participación ciudadana, Salamanca.

\footnotetext{
Abstract

Salamanca was included in the UNESCO World Heritage List 30 years ago. Since then the criteria for managing cultural assets have evolved significantly. In order to update Salamanca's valuable characteristics, a challenging plan is needed based on cultural and environmental aspects. In this context, the City Hall of Salamanca has recently approved the "Green Urban Infrastructure Plan 20202035"(PEPIVB). Through this proposal, a strategy based on a holistic approach has been established in order to integrate the valuable environmental, social, economic and cultural aspects of the city as a whole. This will allow the city to face the new challenges of the 21st century through the implementation of sustainable, long lasting nature based solutions.
}

The strategy is a solid and dynamic plan, able to evolve and adapt to the city's changes and needs. The city will be provided with a consolidating urban tool that is novel in comparison with other green cities. The strategy will be implemented in the whole municipality and is expected to have an impact on the surrounding towns as well as on the entire region. It is an innovative strategy based on two universal values: urban green infrastructure and heritage.

Keywords: urban green infrastructure, world heritage, nature based solutions, urban green network, climate change, ecosystem services, citizen participation, Salamanca. 


\section{La Estrategia Verde en las nuevas directrices de gestión del patrimonio mundial salmantino: el Plan de Gestión de la Ciudad Vieja de Salamanca}

Con el objeto de consolidar el grado de excelencia que ostenta el patrimonio histórico salmantino, el Ayuntamiento de Salamanca aprobó definitivamente en 2017 su Plan de Gestión de la Ciudad Vieja de Salamanca, destinado a preservar sus valores universales excepcionales, que han sido revisados y enriquecidos con una nueva lectura que incorpora todos los aspectos que definen la personalidad e identidad cultural de la ciudad y aspira a mejorar la calidad de vida de sus habitantes en un espacio urbano más sostenible.

En dicho documento ya se indica la necesidad de replantear "la ciudad en su territorio" como su contexto comprensivo y de avanzar en la visión desde diversas escalas (territorio, paisaje lejano, riberas, paisaje cercano, paisajes urbanos... lectura paisajística integradora y estructural). De este modo, dentro de la redefinición de los conceptos del valor universal excepcional de Salamanca se han destacado, entre otros, la calidad ambiental y el paisaje como una de las fortalezas de la ciudad histórica (Fig. 1) que hay que proteger, tanto del territorio, como del entorno o el propio paisaje interior, y en el cual se incluyen los corredores verdes visuales, los hitos de referencia, los miradores y la campiña tradicional circundante, entendidos como elementos integrantes del conjunto monumental que han contribuido a la construcción de la identidad urbana (de la Riva y Vázquez, 2017).

Cabe entender, por tanto, la sintonía de este Plan con visiones más innovadoras del modelo de ciudad que permitan poner en relación los valores culturales del lugar con su dimensión ambiental, manifestándose la necesidad de regular determinados usos y transformaciones urbanas a través de medidas reguladoras para las áreas de protección paisajística.

\section{El Plan Especial de Protección de la Infraestructura Verde Urbana y Biodiversidad 2020-35 (PEPIVB) $^{1}$ : misión, retos y objetivos estratégicos}

Dentro del contexto actual de la dinámica urbana, parece necesario y urgente desarrollar nuevas estrategias que fomenten valores, enfoques y soluciones, preferiblemente basadas en la naturaleza, para renovar nuestros modelos urbanos exhaustos, donde la necesidad de creación/renovación de nuevos espacios y relaciones está siendo cada vez más demandada por la sociedad (Diosdado y Jiménez, 2019).

Este plan o estrategia tiene por objeto la salvaguarda de los valores naturales y el fomento de los servicios de los ecosistémas en el término municipal de una ciudad media, incluida en la Lista del Patrimonio Mundial y ubicada geográficamente en una zona especialmente sensible al cambio climático, para impulsar un nuevo modelo urbano más verde y sostenible que, en coordinación con otros planes estratégicos en marcha (como la EDUSI del Tormes $+^{2,}$ el Plan de Gestión de la Ciudad Vieja, el Plan de Movilidad Urbana Sostenible, Plan de Comunicación de la estrategia de la infraestructura verde con nombre propio: SAVIA RED VERDE SALAMANCA ${ }^{3}$ y el proyecto LIFE19 CCA/ES/001188, Vía de la Plata ${ }^{4}$, orienten el desarrollo y la reconfiguración de la futura ciudad.

\footnotetext{
${ }^{1}$ Este plan ha sido redactado, y coordinado por Beatriz Diosdado Calvo (Patronato Municipal de Vivienda y Urbanismo), con la asistencia técnica de Jorge Ozcáriz Salazar (COMAV -Consultoría Mediambiental Asociados Vitoria S.L), Jaime Díaz Morlán, Oscar Miravalles Quesada e Irene Zúñiga Sagredo (Atalaya Territorio, S.L.), diversos técnicos municipales y la colaboración de la USAL (Universidad de Salamanca). http://infraestructuraverde.aytosalamanca.es/es/plan/documento/ Fecha de consulta: 28/07/20.

${ }^{2}$ Proyecto cofinanciado mediante el programa operativo FEDER de crecimiento sostenible 2014-2020 que supone un perfecto ensamblaje del conjunto río+riberas+barrios a través de proyectos ambientales, sociales y urbanísticos cuya esencia es la regeneración física, económica, ambiental y la inclusión social de las zonas ribereñas desfavorecidas, con un presupuesto total que asciende a 18.900.000 euros y un plazo de ejecución de 5 años (2017-2022) https://www.mastormessalamanca.es/ Fecha de consulta: 25/07/20.

${ }^{3}$ La Estrategia de Infraestructura Verde se pone al servicio de los ciudadanos y su difusión a través de su web: SAVIA RED VERDE SALAMANCA: www.saviasalamanca.es , para mostrar los avances en las acciones y proyectos vinculados al horizonte de la Agenda 2030 y fomentar, a través de ella, la participación e implicación del conjunto de la ciudadanía.

${ }^{4}$ El Patronato Municipal de Vivienda y Urbanismo, del Ayuntamiento de Salamanca, se presentó a la convocatoria del 2019 del programa europeo LIFE (subprogrma cambio climático), siendo seleccionado y aprobado el 5 de junio de 2020 el LIFE19 CCA/ES/001188, Vía de la Plata por la Comisión Europea. Este proyecto desarrolla la estrategia de infraestructura verde de Salamanca, con un presupuesto total cercano a los 3 millones de euros y un plazo de ejecución de 40 meses a partir de septiembre del 2020.
} 
Se trata por tanto, de una estrategia única a implantar en una ciudad Patrimonio de la Humanidad, que ha sido diseñada para establecer una red de infraestructura verde urbana-periurbana en la que se integran por primera vez estos 6 elementos en su gestión: adaptación al cambio climático, infraestructura verde, ciudad patrimonio, servicios de los ecosistemas, inteligencia artificial (Marching Learning) y participación ciudadana, a partir de los tres ejes de la sostenibilidad: Medio Ambiente, Sociedad y Economía, uniendo: biodiversidad + patrimonio + ciudadanía.

Como misión, el Plan Especial de Protección de la Infraestructura Verde y Biodiversidad de Salamanca (Diosdado et al., 2020) plantea:

Establecer una hoja de ruta clara y efectiva para desarrollar actuaciones ambientales que contribuyan a implantar una infraestructura verde en el término municipal, que fije las pautas para la consolidación y/o creación de nuevas áreas verdes, adoptando un paisajismo de carácter mediterráneo que utilice fundamentalmente especies autóctonas. El PEPIVB constituye, en definitiva, un ambicioso proyecto comprometido con la ciudad, con su término municipal, con sus ciudadanos y con el patrimonio cultural, social y natural.

Es un plan VIVO, se adapta a los requisitos actuales, ABIERTO en revisión permanente, PARTICIPATIVO donde la función social cobra cada vez más protagonismo y EVOLUTIVO; debe desarrollarse e ir avanzando según las necesidades y actuaciones programadas.

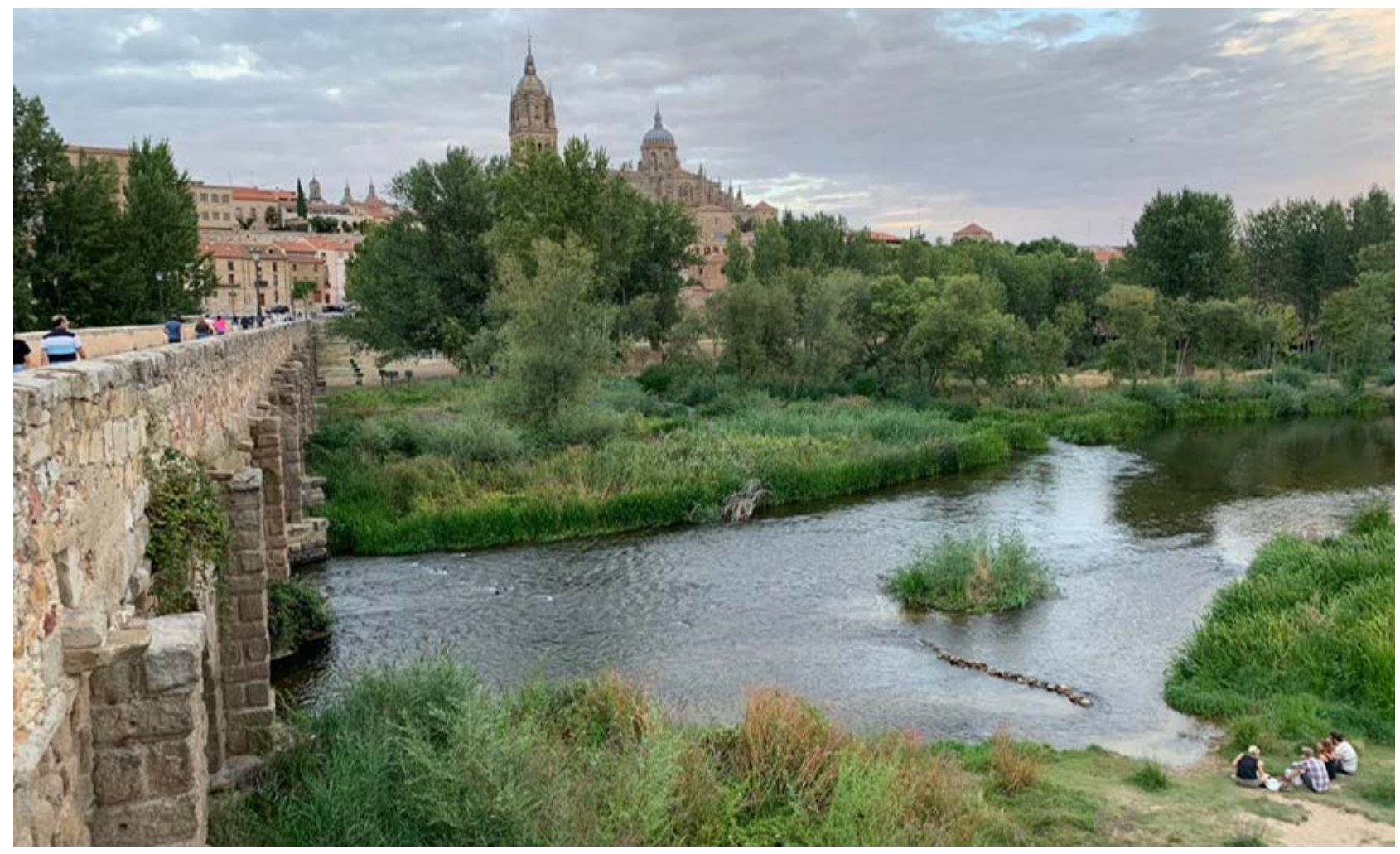

Fuente: Patronato Municipal de Vivienda y Urbanismo (2020)

Fig. 1 Puente romano, ribera río Tormes y catedrales = Biodiversidad + Parimonio + Ciudadanía

Sus retos son: recuperar las conexiones ecológicas, aumentar e introducir más biodiversidad, avanzar hacia la soberanía alimentaria, introducir la dehesa y espacio fluvial en el término, mitigar el cambio climático, avanzar hacia movilidad sostenible, integración del binomio patrimonial cultura-medio ambiente, potenciar el uso social, los beneficios para la salud y hacer un plan participativo y de los salmantinos.

Los objetivos estratégicos son los siguientes:

a) Definir una estructura urbana orgánica, con la progresiva integración, conexión e inclusión de corredores verdes que doten de mayor resiliencia a la ciudad, potenciando la interacción entre la naturaleza y la urbe, así como el disfrute del patrimonio natural, cultural y social por parte de la ciudadanía. 
b) Conectar con las estructuras verdes de municipios colindantes, así como con los ámbitos zonales de corredores verdes (AZCV) propuestos por el Plan Regional del Valle del Duero que unen España con Portugal (PRVDEP), aumentando su superficie y conectividad a través de la Vía de la Plata y demás vías pecuarias que atraviesan Salamanca.

c) Contar con la implicación de la ciudadanía a la hora de implementar el PEPIVB, promoviendo acciones dirigidas a dar a conocer y sensibilizar acerca de los beneficios de la infraestructura verde salmantina e impulsando procesos participativos que contribuyan a mejorar las acciones propuestas en el marco de este Plan.

Mediante la elaboración del PEPIVB se ha conseguido:

- Generar un documento breve, conciso y consensuado por los agentes municipales.

- Diseñar un instrumento eficaz para la transformación ambiental progresiva del conjunto del municipio con incidencia en aquellas partes de la ciudad que más lo necesiten.

- Crear un Plan abierto, en revisión permanente, capaz de adaptarse a las transformaciones inherentes al medio natural y urbano.

En definitiva, esta estrategia constituye la respuesta a los retos de un futuro en el que las poblaciones tendrán que adaptarse a un nuevo paradigma derivado del cambio global que se viene dando en las últimas décadas (Conama, 2018) y, que podría convertirla en un referente transferible a otras ciudades históricas europeas fuertemente pavimentadas.

El documento se ha redactado conforme a la Ley 5/99, de 8 de abril, de Urbanismo de Castilla y León y el Reglamento que la regula, Decreto 22/2004, así como a la Ley 42/2007, de 13 de diciembre, del Patrimonio Natural y la Biodiversidad, está ligado a la Agenda 2030 de la ONU, a la Estrategia de la Comisión Europea de $2014^{5}$ y conforme a los criterios establecidos en la Estrategia Estatal de Infraestructura Verde y de la Conectividad y Restauración Ecológicas (Valladares et al., 2017).

Conceptualmente supera a la red convencional de parques periurbanos, como las existentes en otras ciudades europeas (anillos verdes), con una propuesta innovadora que persigue conectar la ciudad con la naturaleza creando una infraestructura verde que se coordine y complemente con el otro gran valor de Salamanca: su patrimonio histórico. Se trata, por tanto, de poner en valor el capital natural del término municipal con sus distintos ecosistemas; desde el río hasta la dehesa o los corredores ecológicos, creando un paisajismo de carácter meditarráneo que utilice fundamentalmente especies autóctonas y haga un uso más racional de los recursos hídricos.

\subsection{Elementos que lo conforman y su zonificación}

El sistema de infraestrutura verde de Salamanca (IVS) se articula como una red interconectada que incorpora elementos del patrimonio natural y cultural como son los espacios verdes más significativos del ámbito urbano y otros del entorno rural en los que hay mayor presencia de vegetación y actividad agrícola (Fig. 2). La estructura territorial de Salamanca se perfila a través de grandes elementos que tienen que ver con su geomorfología, la red hidrográfica, la cubierta vegetal, los usos del suelo o el paisaje. El río Tormes y el arroyo Zurguén constituyen los dos ejes de agua principales, los cuales, junto a las vías pecuarias que recorren el término municipal y las calles y bulevares arbolados, conforman los principales conectores del sistema. Por ello, partiendo de esta visión territorial se ha realizado una zonificación que integra las distintas clasificaciones urbanas y sus distintos valores.

Cada zona en el ámbito del PEPIVB (Fig. 3) cuenta con vocaciones diferenciadas debido a su clasificación urbana, ya sean suelos rústicos, urbanos o urbanizables y debido a sus valores, ya sean principalmente naturales, patrimoniales o de uso público, siendo posibles sus enlaces a través de puntos de conexión físicos o temáticos (Alario y Macarro, 2017).

\footnotetext{
${ }^{5}$ Communication from the Commission to the European Parlament, the Council, the European Economic and Social Committee and the Committee of the Regions: Green Infrastructure (GI) - Enhancing Europe's Natural Capital (SWD 2013) 155 final.
} 


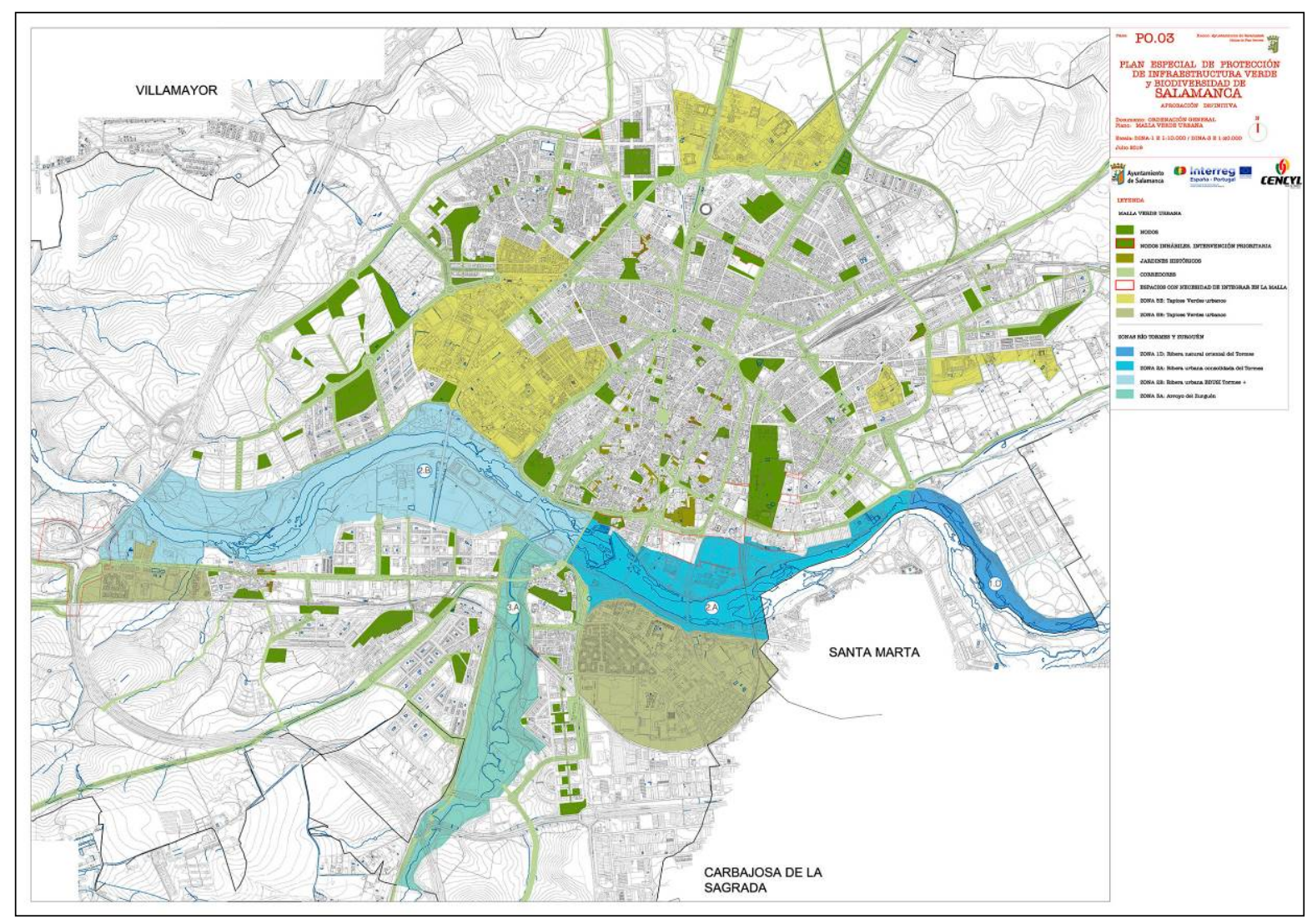

Fuente: Documento memoria justificativa PEPIVB (2020)

Fig. 2 Plano estructura verde en el ámbito urbano del PEPIVB

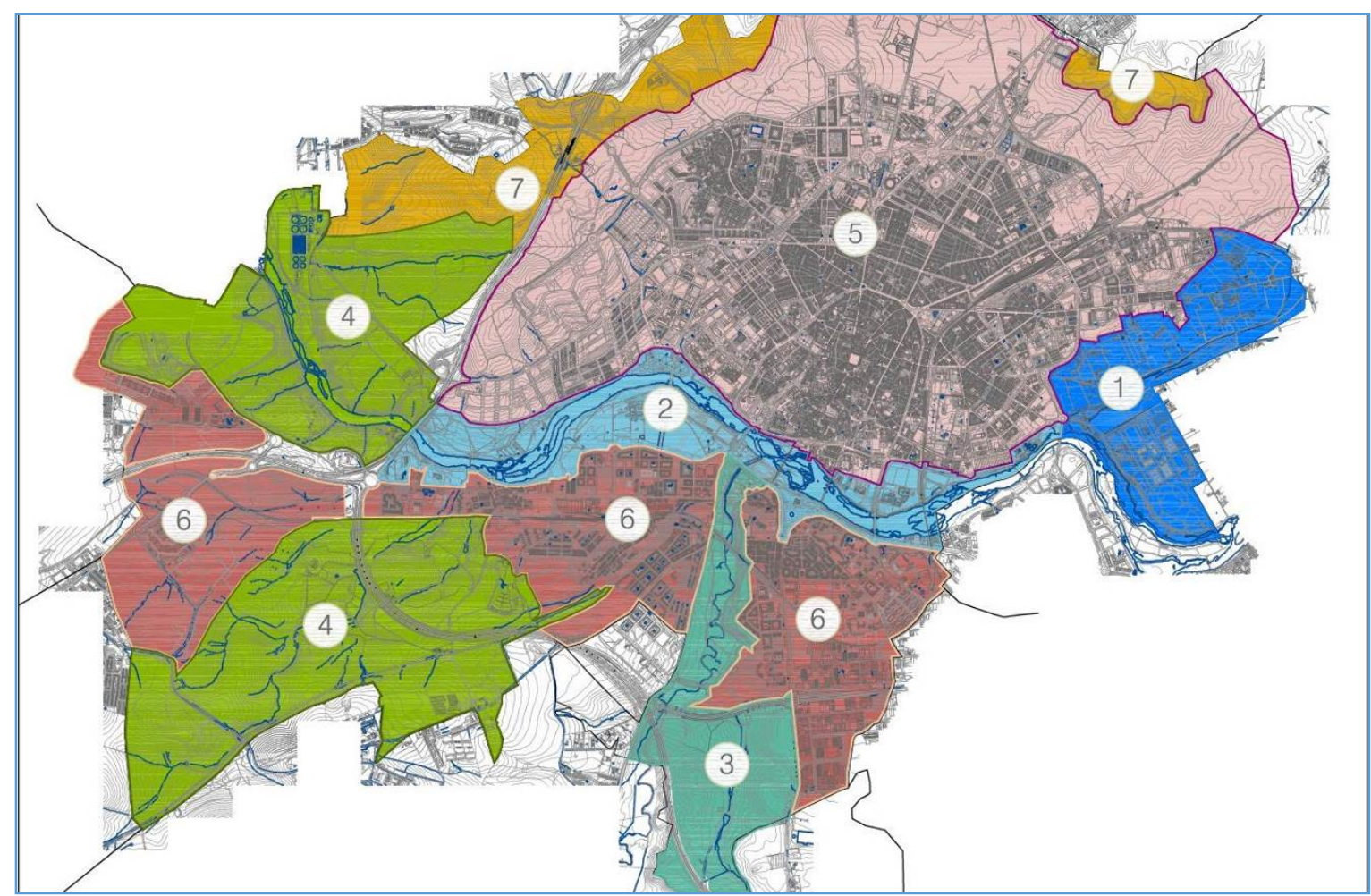

Fuente: Documento memoria justificativa PEPIVB (2020)

Fig. 3 Término municipal de Salamanca: Plano de zonificación 
De esta manera, las zonas $\mathrm{n}^{\mathrm{o}} 1,2,3$ y 4 recogen fundamentalmente los espacios con valores naturales del territorio y sus suelos, o bien se encuentran clasificados como rústicos, o bien son sistemas generales de espacios libres.

Las zonas $\mathrm{n}^{\circ} 5 \mathrm{y} \mathrm{n}^{\circ} 6$ contienen las zonas verdes urbanas y son suelos fundamentalmente urbanos y urbanizables, distinguiéndose los espacios al norte del río Tormes de los del sur. La zona $\mathrm{n}^{\circ} 7$ engloba los terrenos de la corona norte de secano y finalmente, la zona $\mathrm{n}^{\circ} 8$ hace referencia a las conexiones de infraestructura verde que se pueden establecer con los municipios colindantes. Todas estas zonas están vinculadas en su relación con las grandes estructuras territoriales.

\section{Programa General de actuaciones y estudio económico}

Contemplando tanto las variables ecológicas como culturales, el PEPIVB 2020-35 propone una batería de acciones ordenadas temporal y espacialmente, para la reconexión de la ciudad, el territorio y la ciudadanía por medio de una mejora ambiental integral del término municipal, apoyada en sus valores patrimoniales.

El programa de actuaciones se ha elaborado a partir de los objetivos genéricos y las líneas de actuación planteadas, y ha sido categorizado por prioridades de actuación en un marco temporal de 15 años. En primera instancia, debido a la necesidad de implicación de la ciudadanía en el desarrollo del PEPIVB para asegurar su éxito, se han definido unas primeras acciones "estructurales" (de consolidación y proyección) y otras "demostrativas" (de ejecución y de participación ciudadana), la mayor parte de las cuales ya están en marcha y ejecutándose.

El resto del organigrama distingue entre "actuaciones transversales" y "actuaciones por zonas", con un total de 277 acciones propuestas y una previsión económica de gastos de más de 10 millones de euros (para los 5 primeros años) en el lustro, entendiendo que, conforme se recoge en el informe de sostenibilidad económica, la repercusión de los beneficios ecosistémicos sobre el conjunto de la ciudad (de adaptación al cambio climático, mejora de la biodiversidad, mejora de la calidad ambiental, de provisión o abastecimiento, culturales y patrimoniales,..) devolverá sobradamente la inversión realizada en primera instancia.

\section{1. ¿Por dónde se ha empezado?}

El Plan Especial de Protección de Infraestructura Verde y Biodiversidad, se ha aprobado definitivamente por Pleno Municipal el 5 de junio de 2020 (BOCyL nº 130 de fecha 30/06/20). En su previsión de actuaciones a realizar (Tabla 1) y durante su redacción se han puesto en marcha ya las siguientes acciones determinanates que garantizarán su puesta en marcha y desarrollo futuro.

Tabla 1 . Primeras acciones ya realizadas en el PEPIVB 2020-35

\section{Actuaciones estructurales}

\begin{tabular}{|c|c|c|}
\hline \multirow[b]{2}{*}{$\begin{array}{l}\text { De } \\
\text { consolidación }\end{array}$} & $\begin{array}{l}\text { Conformar un Equipo Técnico Municipal responsable del desarrollo y de la gestión del } \\
\text { PEPIVB (PIV_01). }\end{array}$ & \\
\hline & $\begin{array}{l}\text { Habilitar un Presupuesto Municipal Plurianual de mínimos que permita dar visibilidad } \\
\text { al Plan a lo largo de sus primeros años, a completar con fuentes externas de } \\
\text { financiación (PIV_01). }\end{array}$ & Sí \\
\hline \multirow{3}{*}{$\begin{array}{l}\text { De } \\
\text { proyección }\end{array}$} & $\begin{array}{l}\text { Redactar y poner en marcha un Plan de Comunicación, Participación y Promoción del } \\
\text { proyecto (MMI_01). }\end{array}$ & SÍ \\
\hline & $\begin{array}{l}\text { Definir la Red de Vías Verdes del sistema de Infraestructura Verde de Salamanca } \\
\text { (PCMS_01). }\end{array}$ & NO \\
\hline & $\begin{array}{l}\text { Elaborar un Plan de Micropaisajes para la mejora del espacio público en barrios con } \\
\text { déficits de calidad urbana. }\end{array}$ & $\begin{array}{l}\text { (previsión } \\
\text { 2022) }\end{array}$ \\
\hline
\end{tabular}




\section{Actuaciones demostrativas}

Proyectos de mejora de las condiciones ecológicas del río Tormes: regenerar sus $\mathrm{NO}$ riberas, limpiar su cauce, potenciar sus islas como pequeños santuarios de biodiversidad... (1D_01, 2A_02, etc.).

De ejecución

Creación de un eje urbano de infraestructura verde a lo largo de la Vía de la Plata, atravesando el ensanche de la ciudad hasta su salida por el barrio de Pizarrales (5B_05).

Elaboración de una página web, en el marco del Plan de Comunicación, Participación

De y Promoción del PEPIVB (PIV_05): www.saviasalamanca.es

participación ciudadana
Proyecto piloto de embellecimiento de un determinado espacio urbano por los vecinos: jardineras comunitarias, alcorques con flor, concurso de balcones. (previsión

SÍ

SÍ

Fuente: Elaboración propia

\subsection{LIFE19 CCA/ES/001188, Vía de la Plata}

El proyecto LIFE Vía de la Plata, entre septiembre de 2020 y diciembre de 2023 pondrá en marcha la estrategia de la red de infraestructura verde en la ciudad Patrimonio de la Humanidad de Salamanca, convirtiendo la vía pecuaria e itinerario cultural: "Via de la Plata", en un corredor verde a lo largo de 6,9 Km lineales de sur a norte del término municipal, afectando a una superficie total de actuación de $261 \mathrm{Ha}$, divididas en 6 zonas piloto según las diferentes características ambientales y urbanas del término.

Para su desarrollo se llevarán a cabo más de 40 tipos de actuaciones, en su espacio tanto rústico como urbano a través de soluciones basadas en la naturaleza desde el punto de vista de la flora, fauna, gestión del agua, patrimoniales y culturales, unidas todas ellas a través del Learning Maching y el Deep Reinforcement Learning (aprendizaje por refuerzo profundo) con la validación y cuantificación de 46 servicios de los ecosistemas aplicados al proyecto, gracias a los socios: BISITE (Universidad de Salamanca) y la Cátedra Unesco para el Desarrollo Sostenible de la Universidad del Pais Vasco.

A través del mismo se acometerán plantaciones con abundante selección de especies propicias y más eficientes para la adaptación al cambio climático; se crearán calles peatonales con mayor vegetación, alcorques vivos y muros verdes; se promoverán oasis de fauna en determinados jardines de la ciudad y solares; así como la creación de refugios naturales para recuperar especies autóctonas del río Tormes y Arroyo del Zurguén.

En paralelo, se probará una experiencia piloto a través de estaciones de monitorización basadas en sensores tecnológicos e inteligencia artificial para aportar datos, correlaciones, previsiones y recomendaciones frente a eventos y escenarios actuales y futuros en torno a la calidad del aire y usos del suelo, entre otros parámetros. El LIFE Vía de la Plata, llevará a cabo un estudio del estado del arte en tecnologías IoT (Internet of Things - Internet de las Cosas), Edge Computing (computación en el borde la red) y Cloud Computing (computación en la nube) para la captura de datos en soluciones para la monitorización, predicción y mitigación del cambio climático.

\section{Conclusión: Trascendencia de la Estrategia Verde y previsión de resultados}

La implantación paulatina de este Plan novedoso supone un punto de inflexión para la historia de la ciudad y un cambio de modelo urbano y de mentalidad ciudadana acordes con los tiempos que corren en la nueva era del Antropoceno $^{6}$ (Prats, 2017). Mediante la ejecución progresiva de las actuaciones previstas en esta estrategia

\footnotetext{
${ }^{6}$ Estamos viviendo una nueva era geológica denominada Antropoceno, la era del impacto del ser humano sobre la Tierra. Se trata del "periodo histórico donde el volumen de actividades humanas ha tenido tal efecto sobre el planeta, que ha alterado los sistemas fundamentales para el sostenimiento de la vida", caracterizándose por la radical transformación de los ecosistemas terrestres por la actividad humana, llegando a conseguir cambiar el ciclo vital del planeta y sacándolo de su variabilidad natural.
} 
Salamanca, con sus peculiaridades y condicionantes medioambientales, ha optado por apoyarse en la Naturaleza y los beneficios de los servicios de los ecosistemas para garantizar una calidad de vida óptima a sus habitantes en un futuro, en el que su patrimonio cultural de excepción seguirá siendo motor de progreso y símbolo de su identidad urbana. Tal propuesta ya está siendo reconocida internacionalmente tras la reciente aprobación y financiación, por la Comisión Europea, del Proyecto LIFE Vía de la Plata del subprograma, Climate Change Adaptation, cuya implantación será difundida como experiencia a otras ciudades europeas similares en las que el patrimonio histórico tenga un gran peso específico.

A través de los proyectos y acciones adscritas a la estrategia diseñada por el Plan Especial de Protección de la Infraestructura Verde y Biodiversidad, Salamanca creará un nueva ciudad más habitable, apoyándose en la ciudadanía y en un ambicioso plan de difusión y participación -SAVIA SALAMANCA-, cuyo prototipo pueda ser trasladable a otras ciudades históricas con problemáticas medioambientales parecidas y con gran peso en sus patrimonios culturales. Sin duda, un gran reto para esta ciudad, su Administración local y sus ciudadanos.

\section{Referencias}

Alario, C., y Macarro, C. (2017). Guía Arqueológica de Salamanca. En Grupo Ciudades Patrimonio de la Humanidad de España, Ministerio de Educación, Cultura y Deportes (Ed.), Guía Arqueológica de las Ciudades Patrimonio de la Humanidad de España. Fecha consulta: 28/07/20, en http://www.guiaarqueologicaciudadespatrimonio.org/

Atalaya. (2017). Plan Director de la Infraestructura Verde de Zaragoza. Agencia de Medio Ambiente y Sostenibilidad. Zaragoza: Ayuntamiento de Zaragoza. Fecha consulta: 28/07/20, en http://www.zaragoza.es/sede/portal/medioambiente/planinfraverde/

Ayuntamiento de Salamanca. (2016). Estrategia de Desarrollo Urbano Sostenible e Integrado Tormes + (EDUSI Tormes+) 20162022. Documento de candidatura. Salamanca: Ayuntamiento de Salamanca. Fecha consulta: 28/07/20, en https://www.mastormessalamanca.es/pdf/ESTRATEGIA.pdf

Conama. (2018). $14^{\circ}$ Congreso Nacional de Medio Ambiente (26-29 noviembre 2018). Fecha consulta: 28/07/20, en http://www.conama2018.org/web/generico.php?idpaginas=\&lang=es\&menu=370\&id=16\&op=view.

Diosdado, B., y Jiménez, E. (2019). Innovar con la naturaleza, infraestructura verde en Salamanca. En R. Rivero y M. Cerezo Innovación en las normas ambientales (pp. 385-439). Barcelona: Tirant Lo Blanch.

Diosdado, B., COMAV, y Atalaya. (2020). Plan Especial de Protección de la Infraestructura Verde y Biodiversidad de Salamanca. Salamanca: Ayuntamiento de Salamanca. Fecha de consulta: 28/07/20 en http://infraestructuraverde.aytosalamanca.es/es/

Prats, F., et al. (2017). Ante el Antropoceno. Reflexiones sobre la cuestión biorregional en el País Vasco. Departamento de Medio Ambiente, Planificación Territorial y Vivienda. Gobierno Vasco. Fecha consulta 28/07/20, en https://www.euskadi.eus/contenidos/informacion/revision dot/es def/adjuntos/Aprobacion\%20incial/Informe\%20y\%20Anexo Biorregional_Pa\%C3\%ADs\%20Vasco_FPrats.pdf

Riva de la, J. L., Vázquez, G., et al. (2017). Plan de Gestión de la Ciudad Vieja de Salamanca. Salamanca: Ayuntamiento de Salamanca. Junta de Castilla y León. Fecha consulta: 28/07/20, en http://urbanismo.aytosalamanca.es/es/planeamientourbanístico/anuncio_004

Valladares, F., Gil, P., y Forner, A. (2017). Bases científico-técnicas para la Estrategia estatal de la infraestructura verde y la conectividad y restauración ecológicas. Madrid: Ministerio de Agricultura, Pesca, Alimentación y Medio Ambiente. Fecha consulta: 28/07/20, en https://www.miteco.gob.es/es/biodiversidad/temas/ecosistemas-y-conectividad/conectividadfragmentacion-de-habitats-y-restauracion/Infr verde.aspx 